\title{
A mathematical model of kinetochore-microtubule attachment regulated by Aurora A activity gradient describes chromosome oscillation and correction of erroneous attachments
}

\author{
Manuel Alejandro Campos Medina ${ }^{1,2 *}$, Kenji Iemura ${ }^{1}$, Akatsuki Kimura ${ }^{3}$, and Kozo TanakA ${ }^{1 *}$ \\ ${ }^{1}$ Department of Molecular Oncology, Institute of Development, Aging and Cancer, Tohoku University, 4-1 Seiryo-machi, Aoba-ku, \\ Sendai, Miyagi 980-8575, Japan; ${ }^{2}$ Department of Systemic Cell Biology, Max Planck Institute for Molecular Physiology, Otto-Hahn-Str. \\ 11, 44227 Dortmund, Germany; and ${ }^{3}$ Cell Architecture Laboratory, Department of Chromosome Science, National Institute of Genetics; \\ Department of Genetics, The Graduate University for Advanced Studies (SOKENDAI), 1111 Yata, Mishima, Shizuoka 411-8540, Japan \\ (Received 8 July 2021; and accepted 17 July 2021)
}

\begin{abstract}
Chromosome oscillation during metaphase is attenuated in cancer cell lines, concomitant with the reduction of Aurora A activity on kinetochores, which results in reduced mitotic fidelity. To verify the correlation between Aurora A activity, chromosome oscillation, and error correction efficiency, we developed a mathematical model of kinetochore-microtubule dynamics, based on stochastic attachment/detachment events regulated by Aurora A activity gradient centered at spindle poles. The model accurately reproduced the oscillatory movements of chromosomes, which were suppressed not only when Aurora A activity was inhibited, but also when it was upregulated, mimicking the situation in cancer cells. Our simulation also predicted efficient correction of erroneous attachments through chromosome oscillation, which was hampered by both inhibition and upregulation of Aurora A activity. Our model provides a framework to understand the physiological role of chromosome oscillation in the correction of erroneous attachments that is intrinsically related to Aurora A activity.
\end{abstract}

\section{INTRODUCTION}

Most of the cancer cells show aneuploidy, an abnormal number of chromosomes in a cell, which mainly occurs as a result of chromosomal instability (CIN), a condition in which chromosome missegregation occurs at high rates (Bakhoum and Compton, 2012; Gordon et al., 2012; Tanaka and Hirota, 2016). For proper chromosome segregation, kinetochores (KTs) on sister chromatids have to attach to microtubules (MTs) emanating from opposite spindle poles, called bi-orientation (Tanaka et al., 2005; Tanaka, 2013). Erroneous attachments, such as merotelic attachment, where a single KT attaches to MTs from both spindle poles, result in chromosome

*Corresponding authors:

manuel.camposmedina@mpi-drotmund.mpg.de kozo.tanaka.d2@tohoku.ac.jp missegregation and aneuploidy (Cimini, 2008). To avoid chromosome missegregation, there are mechanisms to ensure the establishment of bi-orientation for all the chromosomes. One of the mechanisms is the correction of erroneous attachments by Aurora kinases through phosphorylation of Hec1, a component of the Ndc80 complex that connects KTs directly to MTs, which reduces the affinity of Hec1 to MTs and destabilizes KT-MT attachments (Zaytsev et al., 2014, 2015; Wimbish and DeLuca, 2020). Previous reports suggest that inefficient correction of erroneous KT-MT attachments, which is due to MT stabilization or weakened Aurora B activity, is a cause of CIN in cancer cells (Bakhoum et al., 2009a, 2009b; Abe et al., 2016; Tanaka and Hirota, 2016).

Recently, we reported that inefficient error correction through chromosome oscillation may underlie CIN in cancer cell lines (Iemura et al., 2021). Chro- 
mosome oscillation is an iterative motion of chromosomes around the spindle equator during metaphase (Skibbens et al., 1993; Jaqaman et al., 2010; Wan et al., 2012). We found that chromosome oscillation facilitates $\mathrm{Hec} 1$ phosphorylation, which is mainly dependent on Aurora A on the spindle (Iemura et al., 2021). In the opposite way, Hecl phosphorylation by Aurora A is required for efficient chromosome oscillation (DeLuca et al., 2018; Iemura et al., 2021). Furthermore, enhancement of chromosome oscillation reduced the number of erroneous KT-MT attachments and chromosome missegregation, whereas Aurora A inhibition during metaphase increased these errors. Chromosome oscillation and $\mathrm{Hecl}$ phosphorylation during metaphase are attenuated in cancer cell lines, which may be a cause of CIN. Our data suggest that poleward chromosome motion during oscillation increases the chance of $\mathrm{Hec} 1$ phosphorylation on KTs due to the presence of a declining Aurora A gradient along the spindle axis, from spindle poles to the spindle equator, destabilizing KT-MT attachments and promoting the correction of erroneous KT-MT attachments (Fig. 1). However, the molecular and biophysical basis for this unprecedented correlation between Aurora A activity, chromosome oscillation, and error correction efficiency, is not well-understood. In addition, it is unclear why chromosome oscillation is attenuated in cancer cells, even though it is known that Aurora A is upregulated in various cancer cells (Farag, 2011).

Chromosome motion during mitosis depends on the forces acting on a chromosome, which is a dynamic process difficult to understand quantitatively in detail due to its complexity. Mathematical modelling has been contributing to the quantitative understanding of the mitotic chromosome dynamics including chromosome oscillation (Civelekoglu-Scholey and Cimini, 2014). To get insights into the role of the Aurora A activity gradient on chromosome oscillation and correction of erroneous KT-MT attachments, we developed a mathematical model based on previous models by incorporating the Aurora A activity gradient as a factor that affects Hecl affinity to MTs.

\section{MATERIALS AND METHODS}

Cell culture and synchronization. RPE-1 cells (human retinal pigment epithelial cells, a gift from $\mathrm{H}$. Hochegger) and HeLa Kyoto cells (human cervical carcinoma cells, a gift from T. Hirota) were grown at $37^{\circ} \mathrm{C}$ in a $5 \% \mathrm{CO}_{2}$ atmosphere in Dulbecco's modified Eagle's medium (Nacalai Tesque), supple- mented with $10 \%$ fetal bovine serum. Cells were synchronized to metaphase by $20 \mu \mathrm{M}$ MG132 for $2 \mathrm{~h}$. To inhibit Aurora A, synchronized cells were treated with $50 \mathrm{nM}$ of MLN8237 (Cayman Chemicals) for $1 \mathrm{~h}$.

Live cell imaging. RPE-1 cells and HeLa cells expressing EGFP- $\alpha$-tubulin and EGFP-CENP-A were grown in glass chambers (Thermo Fisher Scientific). One hour before imaging, the medium was changed to pre-warmed Leibovitz's L-15 medium (Thermo Fisher Scientific) supplemented with $20 \%$ fetal bovine serum and $20 \mathrm{mM}$ HEPES, pH 7.0. Recordings were made in a temperature-controlled incubator at $37^{\circ} \mathrm{C}$. Z-series of five sections in $0.5 \mu \mathrm{m}$ increments were captured every 2 seconds. Image stacks were projected with three Z-sections and used for tracking. KT positions and spindle edges were tracked in deconvolved movies using the StackReg (Thevenaz et al., 1998) and Manual Tracking (http://rsb.info. nih.gov/ij/plugins/track/track.html) plug-ins for Fiji (Schindelin et al., 2012). KT to equator distance was normalized by spindle length at each time point according to the previous study (Iemura and Tanaka, 2015). All time-lapse images were collected with an Olympus IX-71 inverted microscope controlled by DeltaVision softWoRx using a $\times 1001.40$ NA Plan Apochromat oil objective lens.

A force-balance model describing chromosome $\mathrm{ki}$ netics. In order to explore the probable roles of Aurora $\mathrm{A}$ on the chromosome motion, and establish an error correction mechanism based on it, we modified a model developed to describe the chromosome kinetics in Ptk1 cells (Civelekoglu-Scholey et al., 2013). The interactions between KTs and MTs are measured by the amount of the Ndc 80 complexes, a protein structure located at the tip of KTs. Similar to another stochastic model (Gay et al., 2012), the Ndc80 complexes are modelled to allow 2 possible states, attached to a MT or detached. The affinity between KTs and MTs is described by a mathematical expression that depends on the distance between the chromatid and its corresponding spindle pole. The chromosome poleward motion is centered on the spindle poles; their pulling force $\left(F_{\text {pull }}\right)$ depends on the speed of the attached KT (Fig. 2A; the definitions of the parameters hereafter are listed in Table 1-4). This is counterbalanced by the cohesive force $\left(F_{c o h}\right)$, the representation of the cohesive bonds between both chromatids, the position-dependent polar ejection force $\left(F_{P E}\right)$, and the velocity-dependent drag force $\left(F_{d r a g}^{R K T}\right)$ of the chromatids (Fig. 2A). 


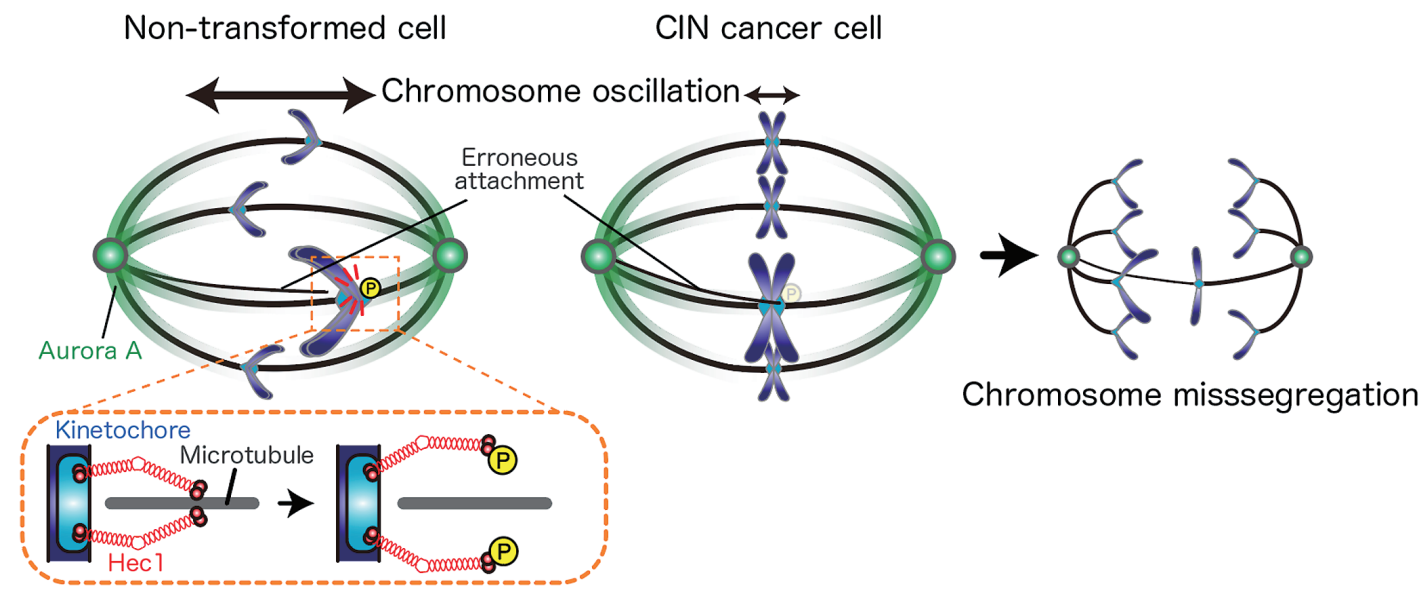

Fig. 1 Chromosome oscillation and correction of erroneous KT-MT attachments in non-transformed and CIN cancer cells. On the metaphase spindle, Hec1 on kinetochore (KT) is phosphorylated by Aurora A, which distributes on the spindle as a gradient from a pole to the equator, when KT moves towards a pole through chromosome oscillation. Hec1 phosphorylation by Aurora A reduces the affinity of KT to microtubules (MTs), which is required for correction of erroneous KT-MT attachments as well as efficient chromosome oscillation. In cancer cells that exhibit chromosomal instability (CIN), chromosome oscillation is attenuated, which leads to reduced Hec1 phosphorylation and inefficient correction of erroneous attachments, resulting in the occurrence of chromosome missegregation. P: phosphorylation.

A

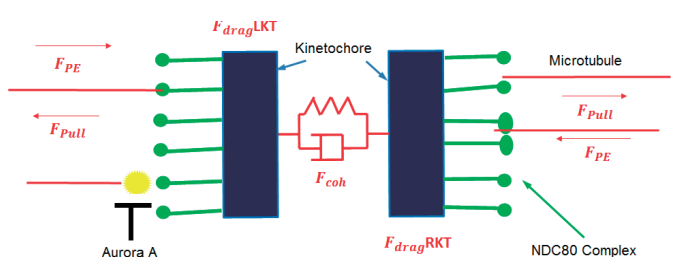

C

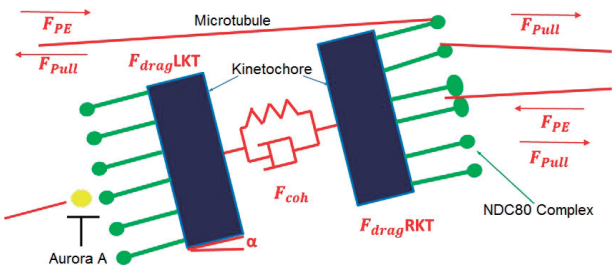

$\mathrm{E}$

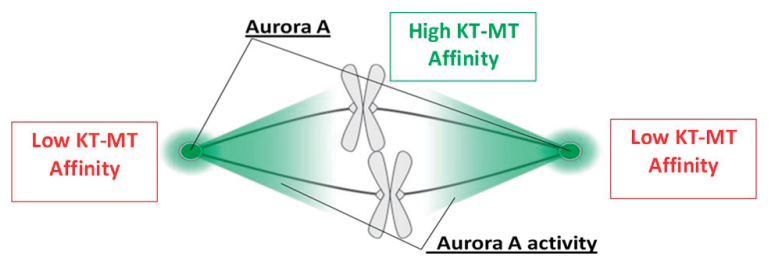

B

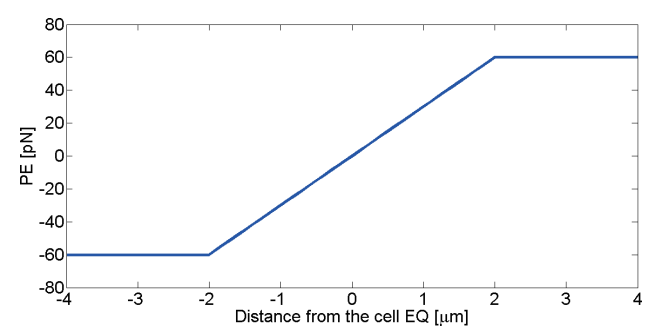

D

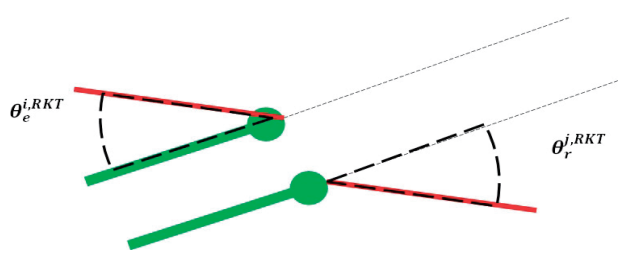

$\mathrm{F}$

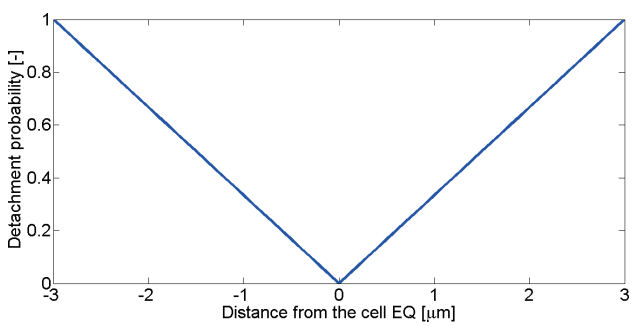

Fig. 2 A force-balance model describing chromosome kinetics. (A) A uni-dimensional free body diagram depicting the forces exerted on sister KTs. (B) Polar ejection force distribution in the spindle. (C) A bi-dimensional free body diagram. (D) Close up of a Ndc80-MT interaction for proper and erroneous attachments in the bi-dimensional interpretation. (E) A schematic diagram of the Aurora A-like activity gradient on the spindle. (F) A mathematical expression of the Aurora A-like activity gradient. 
Table 1 Structure dependent parameters overview

\begin{tabular}{|c|c|c|c|}
\hline Symbol & Definition & $\begin{array}{l}\text { Value } \\
\text { [unit] }\end{array}$ & Reference \\
\hline$N_{K T}$ & $\begin{array}{c}\text { Maximal number of MT attach- } \\
\text { ment sites at KT }\end{array}$ & $30[-]$ & $\begin{array}{c}\text { (Civelekoglu-Scholey et al., } \\
\text { 2013) }\end{array}$ \\
\hline$N_{N D C 80}^{M A X}$ & $\begin{array}{c}\text { Maximal number of Ndc80 } \\
\text { complexes attachment sites at } \\
\text { KT }\end{array}$ & $13[-]$ & $\begin{array}{c}\text { (Civelekoglu-Scholey et al., } \\
\text { 2013) }\end{array}$ \\
\hline$N_{\text {slid }}$ & $\begin{array}{c}\text { Number of sliding motors per } \\
\text { attached KT }\end{array}$ & $4[-]$ & $\begin{array}{c}\text { (Civelekoglu-Scholey et al., } \\
\text { 2013) }\end{array}$ \\
\hline
\end{tabular}

Table 2 Mechanical and biophysical parameters overview

\begin{tabular}{|c|c|c|c|}
\hline Symbol & Definition & $\begin{array}{l}\text { Value } \\
\text { [unit] }\end{array}$ & Reference \\
\hline$d_{o}^{c o h}$ & Rest length of cohesive bonds & $1.5[\mu \mathrm{m}]$ & $\begin{array}{c}\text { (Civelekoglu-Scholey et al., } \\
\text { 2013) }\end{array}$ \\
\hline$L_{\text {cell }}$ & Cell length & $14[\mu \mathrm{m}]$ & (Goshima and Scholey, 2010) \\
\hline$k_{c o h}$ & $\begin{array}{c}\text { Spring constant of cohesive } \\
\text { bonds }\end{array}$ & $50\left[\frac{\mathrm{pN}}{\mu \mathrm{m}}\right]$ & $\begin{array}{c}\text { (Civelekoglu-Scholey et al., } \\
\text { 2013) }\end{array}$ \\
\hline$\mu_{C h r}$ & $\begin{array}{c}\text { Viscous drag coefficients of } \\
\text { chromosome }\end{array}$ & $10\left[\frac{\mathrm{pN}}{\mu \mathrm{m} \mathrm{s}}\right]$ & $\begin{array}{c}\text { (Civelekoglu-Scholey et al., } \\
\text { 2013) }\end{array}$ \\
\hline$\epsilon_{c o h}$ & $\begin{array}{l}\text { Viscous friction constant of } \\
\text { cohesive bonds }\end{array}$ & $50\left[\frac{\mathrm{pN}}{\mu \mathrm{m} \mathrm{s}}\right]$ & $\begin{array}{c}\text { (Civelekoglu-Scholey et al., } \\
\text { 2013) }\end{array}$ \\
\hline$V_{\text {Slid }}^{\text {MAX }}$ & $\begin{array}{l}\text { Unloaded velocity of sliding } \\
\text { motors }\end{array}$ & $2.5\left[\frac{\mu}{\min }\right]$ & $\begin{array}{l}\text { Estimated from (Hong et al., } \\
\text { 2016) }\end{array}$ \\
\hline$F_{\text {Slid }}^{\text {STALL }}$ & Stall force of sliding motors & $5[\mathrm{pN}]$ & $\begin{array}{l}\text { Estimated from (Hong et al., } \\
\text { 2016) }\end{array}$ \\
\hline$F_{P E}^{M A X}$ & $\begin{array}{l}\text { Polar ejection force at the } \\
\text { spindle poles }\end{array}$ & $30[\mathrm{pN}]$ & $\begin{array}{c}\text { Estimated from (Brouhard and } \\
\text { Hunt, 2005) }\end{array}$ \\
\hline
\end{tabular}

The model is based on the following equation system. The cohesive force, modelled as a buffered Hooke's spring, is given by the following expression that depends on the position and speed of the chromatids:

$$
F_{c o h}=k_{c o h}\left(X_{K T}^{R}-X_{K T}^{L}-d_{0}^{c o h}\right)-\epsilon_{c o h}\left(v_{K T}^{R}-v_{K T}^{L}\right)
$$

Placing the system of reference on the right KT, the viscous stress, caused by the motion of the chromosome through the media, is described as follows:

$$
F_{d r a g}^{R K T}=\mu_{c h r} v_{K T}^{R}
$$

Considering the $m$-th MT attached to the right KT, with an affinity defined by the variable $A_{f}$ (ratio of the attached Ndc80 complexes over maximum allowed attachments), the force generated at the spindle pole depends on the speed of the attached chromatid, and is given by the following expression:

$$
\begin{gathered}
F_{\text {pull }}^{m}=F_{\text {slid }}^{\text {Stall }} N_{\text {slid }} A_{f}\left(1-\frac{v_{K T}^{R}}{v_{\text {slid }}^{\max }}\right) \\
F_{\text {pull }}^{R K T}=\sum_{\mathrm{m}=1}^{\# k M T} F_{\text {pull }}^{m}
\end{gathered}
$$

For the erroneous attachment, based on the sign convention, the affinity factor has a negative value.

The polar ejection forces $\left(F_{P E}\right)$ vary according to the distance between the KT and the attached spindle pole, based on the previous study ( $\mathrm{Ke}$ et al., 2009). Their distribution is shown in Fig. 2B.

Considering that the system is in mechanic equilibrium, the balance is as follows:

$$
F_{d r a g}^{R K T}=F_{R K T}-F_{c o h}-F_{P E}
$$

The expressions for the left KT are analogous to the ones presented above.

This model conceives the Ndc80 complex as a rigid body, whereas the inter-KT space is described by a buffered Hooke's spring. The system moves ac- 
Table 3 Kinetic parameters overview

\begin{tabular}{|c|c|c|c|}
\hline Symbol & Definition & $\begin{array}{l}\text { Value } \\
\text { [unit] }\end{array}$ & Reference \\
\hline$k_{c a t}$ & MT plus end catastrophe rate & $0.01\left[\frac{1}{\mathrm{~s}}\right]$ & (Kinoshita et al., 2001) \\
\hline$P_{\text {erroneous }}$ & $\begin{array}{l}\text { Erroneous attachment occur- } \\
\text { rence rate (1D) }\end{array}$ & $0.1[-]$ & Assumed in this study \\
\hline$\pi_{A U R A}$ & $\begin{array}{l}\text { Aurora kinase A-like activity } \\
\text { factor }\end{array}$ & $4[-]$ & Estimated in this study \\
\hline$\Delta \mathrm{t}$ & Time step & $0.1[\mathrm{~s}]$ & Assumed in this study \\
\hline$\tau$ & $\begin{array}{l}\text { Pre-exponential adjustment } \\
\text { constant }\end{array}$ & $4[-]$ & $\begin{array}{l}\text { Estimated based on the obser- } \\
\text { vations of (Long et al., 2017) }\end{array}$ \\
\hline$\delta$ & $\begin{array}{l}\text { Exponential coefficient adjust- } \\
\text { ment constant }\end{array}$ & $4[-]$ & $\begin{array}{l}\text { Estimated based on the obser- } \\
\text { vations of (Long et al., 2017) }\end{array}$ \\
\hline$A u r_{A o}$ & $\begin{array}{c}\text { Baseline associated Aurora A } \\
\text { expression level constant }\end{array}$ & {$[-]$} & Estimated in this study \\
\hline
\end{tabular}

Table 4 Main variables overview

\begin{tabular}{|c|c|c|}
\hline Symbol & Definition & Unit \\
\hline$v x_{K T}^{R} / v x_{K T}^{L}$ & $\begin{array}{l}\text { Horizontal velocity of the right/left } \\
\text { position }\end{array}$ & $\frac{\mu \mathrm{m}}{\mathrm{s}}$ \\
\hline$v y_{K T}^{R} / v y_{K T}^{L}$ & $\begin{array}{l}\text { Vertical velocity of the right/left } \\
\text { position }\end{array}$ & $\frac{\mu \mathrm{m}}{\mathrm{s}}$ \\
\hline$X_{K T}^{R} / X_{K T}^{L}$ & $\begin{array}{c}\text { Horizontal position of the right/left } \\
\text { chromatid }\end{array}$ & $\mu \mathrm{m}$ \\
\hline$Y_{K T}^{R} / Y_{K T}^{L}$ & $\begin{array}{l}\text { Vertical position of the right/left } \\
\text { chromatid }\end{array}$ & $\mu \mathrm{m}$ \\
\hline$P_{d}$ & Detachment probability & - \\
\hline$P_{a}$ & Attachment probability & - \\
\hline$P_{\text {erroneous }}$ & $\begin{array}{l}\text { Erroneous attachment occurrence } \\
\text { probability (2D) }\end{array}$ & - \\
\hline$F_{\text {pull }}$ & Pulling force conveyed by the kMTs & $\mathrm{pN}$ \\
\hline$F_{c o h}$ & Cohesive force & $\mathrm{pN}$ \\
\hline$F_{P E}$ & Polar ejection force & $\mathrm{pN}$ \\
\hline$F_{K T}^{d r a g}$ & Drag force on the chromatid & $\mathrm{pN}$ \\
\hline$A_{f}$ & Affinity factor & - \\
\hline $\operatorname{Aur}_{A}$ & Aurora A expression level parameter & - \\
\hline$N_{N D C 80}$ & $\begin{array}{l}\text { Number of connected Ndc80 complex- } \\
\text { es }\end{array}$ & - \\
\hline$F_{g}$ & $\begin{array}{l}\text { Aurora A-dependent, strength gradient } \\
\text { between shrinking and growing KT }\end{array}$ & - \\
\hline$\beta$ & $\begin{array}{l}\text { Erroneous attachment occurrence rate } \\
\qquad \text { (2D) }\end{array}$ & - \\
\hline$\alpha$ & $\begin{array}{l}\text { Angle between the chromosome axis } \\
\text { and the spindle axis }\end{array}$ & - \\
\hline
\end{tabular}


cording to the affinity gradient between the chromatids. Coincidently, the affinity decreases as the chromatids approach the spindle poles. Therefore, once the affinity of the leading KT is surpassed by the trailing KT, the system changes direction. Despite, each MT binds to a KT by several Ndc80 complexes through a switch-like on-off mechanism, the states of these connections are independent from each other. All these states are stochastically set by new attachments, catastrophe events, or Aurora A-driven detachments. It should be taken into account that the latter holds a rate of occurrence inversely proportional to the distance between the chromatid and its correspondent kinetochore.

A model including sister chromatids rotation (bi-dimensional (2D) interpretation). In the model described above, sister chromatids only move along the spindle axis (uni-dimensional (1D) interpretation). As the erroneous attachment occurrence rate is set arbitrarily in this modeling, the results will thus depend strongly on the arbitrary value. To bypass this effect and allow the erroneous attachments to occur according to a mathematical relationship intrinsic to the model, we included rotation of the sister chromatids to the simulation (bi-dimensional (2D) interpretation). This consists of 2 extra degrees of freedom, rotation and vertical translation of the chromatids (Fig. 2C), to the core equations presented in the previous sections.

The main modification of this interpretation is the inclusion of the angle $\alpha$, which has to be considered in the equations of the model in the form of the polar to Cartesian coordinates linear transformation $[\cos (\alpha) \hat{x}+\sin (\alpha) \hat{y}]$. Consequently, the 2 coupled velocities (right KT and left KT) become 4, which corresponds to the vertical and horizontal translation for each KT. In addition, the force conveyed by the bound MTs becomes dependent on the angle formed between the axis of the MT and the KT. Looking at a close-up of one of the hypothetical attachments presented in Fig. 2C, we can define the angles for the erroneous attachment (denoted as $i$ ) to the Ndc80 complex on the right KT from the left spindle pole, and the proper attachment (denoted as $j$ ) from the right spindle pole (Fig. 2D). These angles are then projected on the axis of the attachment site, which is by definition $\alpha$, by a similar polar to Cartesian coordinates linear transformation $\left[\cos \left(\theta_{r}^{j, R K T}\right)\right.$ $\left.\widehat{x_{\alpha}}+\sin \left(\theta_{r}^{j, R K T}\right) \widehat{y_{\alpha}}\right]$. However, it must be taken into account that the angles formed by the erroneous, and correct attachments are specific for each KT. These projections are transformed into the regular
Cartesian coordinates and included in the equations. Finally, we defined a factor $\beta$; as the mathematical expression that yields the occurrence rate of erroneous attachments according to the bi-orientability of the chromosome (Gay et al., 2012). This function depends on the vertical position of the chromosomes, and is defined in the next section.

Main simplifying assumptions and its consequences. We assume that the amount of proper (kMTs), and erroneous attachments (ekMTs) are set randomly, at time $\mathrm{t}=0$. The $\mathrm{kMT}$ plus end and the attachment site at the Ndc80 complex are modelled as a unit, in other words, the tip of an attached MT moves with the KT it is linked to. The MTs whose plus ends are not attached are not considered. The attachment and detachment of MT are stochastically set. The minus ends of the kMTs are depolymerized at the same speed as the chromatids are moving towards the spindle poles. We neglect the polymerization ratcheting forces that could be exerted at the inner KT plate. The motor proteins at the spindle poles are set to generate force according to the velocity of the chromatid. MTs shrink or grow, according to the poleward or anti-poleward motion of its attachment site. The amount of force a MT transmits to the KT is directly related to its amount of the Ndc80 complexes attached (affinity), its state (shrinking or growing) (Asbury, 2017), and the speed of the chromatid (Dumont and Mitchison, 2009). Furthermore, based on recent findings regarding the effect of the phosphorylation of the Hecl complex by Aurora kinases during metaphase, we adjusted our model to couple appropriately to either polymerizing or depolymerizing MTs (Long et al., 2017). The plus ends of the MTs are modelled as a uniform distribution. Based on the fact that this study focuses on middle chromosomes, the polar ejection forces are considered to vary solely to the horizontal position of the chromatid. Aurora A destabilizes the affinity of the KT-MT attachments as a function dependent on the distance between the chromatid and its correspondent spindle pole (Fig. 2E). Based on a geometrical simplification, the erroneous attachments are considered to have a maximum affinity that corresponds to half of a proper attachment. In addition, the rate of occurrence of the erroneous attachments is considered as a constant for the 1D interpretation, and a variable in the $2 \mathrm{D}$ interpretation depending on the bi-orientability of the chromosome towards the spindle poles.

This model was constructed from a minimal set of assumptions. However, in order to reproduce the 
experimental results observed in different types and conditions of the cells, this set was gradually increased (Table 5). Using the mathematical expressions as described above, a system of coupled equations to find 2 or 4 (1D or $2 \mathrm{D}$ ) velocities was constructed. The system was solved using a built-in Powell dog leg method and an ad hoc explicit Euler algorithm, by a custom-made MATLAB script.

Numerical solution algorithm. Both 1D and 2D interpretations of the model are solved using the same algorithm. The position of the KTs and the attached MTs are calculated by Euler forward method, using ad hoc MATLAB scripts. The attachment-detachment dynamics of correct and erroneous attachments are determined by stochastic mathematical expressions. For the 1D interpretation, the initial conditions are set as follows: the sister chromatids are placed at the equator of the cell in their respective resting position $\left[X_{K T}^{R}(0)=\frac{d_{o}^{\text {coh }}}{2}, X_{K T}^{L}(0)=-\frac{d_{o}^{\text {coh }}}{2}\right]$; the MT minus ends are positioned on the spindle poles, whereas the plus ends are positioned close to the KTs. The state of the MTs (normally attached/erroneously attached/detached) is randomly selected. None of the MTs are attached to either of the KTs, thus all of the forces are equal to zero. Ergo, the system is at rest.

For the 2D interpretation, the sister chromatids are placed at the equator of the cell in their respective horizontal and vertical resting position. For the right $\mathrm{KT}\left[X_{K T}^{R}(0)=\frac{d_{o}^{\text {coh }} \cos (\alpha)}{2}, Y_{K T}^{R}(0)=\frac{d_{o}^{\text {coh }} \sin (\alpha)}{2}\right]$, for the left KT $\left[X_{K T}^{L}(0)=\frac{-d_{o}^{\text {coh }} \cos (\alpha)}{2}, Y_{K T}^{L}(0)=\frac{-d_{o}^{\text {coh }} \sin (\alpha)}{2}\right]$. The angle $\alpha$, as presented in Fig. $2 \mathrm{C}$, represents the angle between the spindle axis and the chromosome. This angle was set at $\frac{\pi}{2}$ to evaluate the error correction mechanism efficiency, but it can take values in the range $\left[0, \frac{\pi}{2}\right]$. The MT minus ends are positioned on the spindle poles whereas the plus ends are positioned close to the KTs. The state of the MTs (normally attached/erroneously attached/detached) is randomly selected. None of the MTs are attached to either of the KTs, thus all of the forces are equal to zero. Analogously to the 1D interpretation, the system is at rest.

Once the simulation starts, the attached/detached statuses of the MTs are set by comparing a randomly-generated number $M$ to the position dependent detachment/attachment probability $P_{d} / P_{a}$. During this step, the status of the hypothetical attachment, normal or erroneous, is also set. A number $N$ is randomly generated and compared to the corresponding probability $P_{\text {erroneous. }}$ This is a fixed value for the 1D interpretation, and a mathematical expression that depends on the vertical position of the KTs for the $2 \mathrm{D}$ interpretation $\left(\beta(t)=\frac{1}{2} \frac{Y_{K T}^{R}(t)-Y_{K T}^{L}(t)}{d_{o}^{\text {cot }}}\right)$. If the value is bigger than the probability of an erroneous attachment to happen, the attachment is ruled as normal. Next, the catastrophe event probability is calculated by an analogous method, a randomly-generated number $O$ is compared to the corresponding value $P_{c a t}$. Once the MT statuses have been updated, the force-balanced nonlinear equation system is solved via Powell's dog leg method, to find the new velocities of the KTs. These velocities are, coincidently, the velocities of the corresponding MTs. Then, the positions of the KTs and MTs are updated, following the previously stated numerical method. In other words, $X_{K T}^{R}\left(t_{\mathrm{m}+1}\right)=X_{K T}^{R}\left(t_{m}\right)+v_{K T}^{R} \Delta t(1 \mathrm{D})$ and $\left[X_{K T}^{R}\right.$ $\left(t_{\mathrm{m}+1}\right)=X_{K T}^{R}\left(t_{m}\right)+v x_{K T}^{R} \Delta t, Y_{K T}^{R}\left(t_{\mathrm{m}+1}\right)=Y_{K T}^{R}\left(t_{m}\right)+v y_{K T}^{R}$ $\Delta t]$ (2D). Finally, the time is increased with the established step, $t_{\mathrm{m}+1}=t_{m}+\Delta t$, which sets up the initial conditions for the next iteration. This algorithm was repeated in the equivalent of several hours in real time, to ensure the stability of the system.

MT-attachment mechanism. MTs connect to a KT when 2 conditions are fulfilled. These are set by 2 independent mechanisms. The first one depends on a stochastic test; a randomly-generated number is compared to a constant (attachment probability) $P_{a}$. If this test is successful, another random number is generated, and compared to the complement of the detachment probability $\left(1-P_{d}\right)$, which is a position-dependent function (Aurora A interpretation). The constant $P_{a}$ is calculated by the following mathematical expression:

$$
P_{a}=1-e^{-A t r \Delta t}
$$

$P_{a}=$ Attachment probability

Attr $=$ attachment rate

$\Delta t=$ Time step of the simulation

Aurora A-like activity gradient. Our interpretation of destabilization of the KT-MT interaction caused by Aurora A is modelled in the form of a linear equation with the minimum value (highest stability) at the spindle equator and the maximum value (lowest affinity) between this point and the spindle pole. This behavior and the parameters used to describe it 
Table 5 Assumptions and simplifications overview

\begin{tabular}{|c|c|c|}
\hline Main assumptions & Added Assumptions & $\begin{array}{l}\text { Assumptions necessary to reproduce the } \\
\text { experimental observations }\end{array}$ \\
\hline $\begin{array}{l}\text { KT-bound MTs undergo directional in- } \\
\text { stability }\end{array}$ & $\begin{array}{l}\text { The separation between each attachment } \\
\text { site on the kinetochore is neglected }\end{array}$ & $\begin{array}{l}\text { The affinity of the KT-MT interactions } \\
\text { depends on the dynamic state of the MT }\end{array}$ \\
\hline $\begin{array}{l}\text { The depolymerization rate of the MT at } \\
\text { the minus end is equal to the speed of } \\
\text { the chromatid }\end{array}$ & $\begin{array}{l}\text { MT plus end attachments and catastro- } \\
\text { phe events occur stochastically }\end{array}$ & $\begin{array}{l}\text { The affinity of the erroneous KT-MT in- } \\
\text { teractions is limited to a } 50 \% \text { of a prop- } \\
\text { er attachment }\end{array}$ \\
\hline $\begin{array}{l}\text { The dynamic state of the MTs is dictat- } \\
\text { ed by its attachment site }\end{array}$ & $\begin{array}{l}\text { Aurora A-dependent detachment of KT- } \\
\text { bound MT is pole-distance dependent } \\
\text { (Increase as the distance decreases) }\end{array}$ & $\begin{array}{l}\text { Depolymerizing and polymerizing MTs } \\
\text { are differentially coupled to KT, accord- } \\
\text { ing to the Hec } 1 \text { phosphorylation level }\end{array}$ \\
\hline $\begin{array}{l}\text { The interkinetochore space behaves as a } \\
\text { viscoelastic material }\end{array}$ & $\begin{array}{l}\text { The erroneous attachments are deter- } \\
\text { mined stochastically, at a constant rate } \\
\text { on the uni-dimensional case, and based } \\
\text { on a mathematically defined relationship } \\
\text { on the bi-dimensional case }\end{array}$ & $\begin{array}{l}\text { The polar ejection force depends solely } \\
\text { on the horizontal position of the chro- } \\
\text { mosome }\end{array}$ \\
\hline $\begin{array}{l}\text { Once attached, the MTs exert a pole- } \\
\text { ward or anti-poleward force, based on } \\
\text { the core equations of the model }\end{array}$ & $\begin{array}{l}\text { On the bi-dimensional case, the attach- } \\
\text { ment angle between the bound MT and } \\
\text { the KTs axis affects the force conveyed } \\
\text { to the chromatid }\end{array}$ & \\
\hline \multicolumn{3}{|l|}{$\begin{array}{l}\text { Ndc } 80 \text { complexes and KTs behave as } \\
\text { rigid bodies }\end{array}$} \\
\hline \multicolumn{3}{|l|}{$\begin{array}{l}\text { Only bound MTs are considered in the } \\
\text { simulation }\end{array}$} \\
\hline $\begin{array}{l}\text { The attachment and detachments that } \\
\text { occur at each Ndc } 80 \text { are independent }\end{array}$ & & \\
\hline
\end{tabular}

were estimated by comparing the chromosome dynamics of the simulation with experimental observations. In this research, a change in the Aurora A expression level implies a change in the slope of the expression, which is defined as follows (Fig. 2F):

$$
P_{d}=A u r_{A} X_{K T}^{R}(t)
$$

$X_{K T}^{R}=$ Position of the right $\mathrm{KT}$ at the time $\mathrm{t}$

$P_{d}=$ Detachment probability

Aur $_{A}=$ Aurora A expression level parameter

Despite the above presented expression is defined for the right $\mathrm{KT}$, there is an analogous equation that describes the detachment probability that corresponds to the left KT.

Effect of Hecl phosphorylation and shrinking-growing affinity gradient. Based on former researches, we are considering that the shrinking MTs (bound to poleward motion) have a high affinity to the KTs. In contrast, the growing MTs (bound to anti-poleward motion) have a low affinity to the KTs. Additionally, as it has been reported recently (Long et al., 2017), the Hecl phosphorylation is intrinsically related to the affinity between anti-poleward motion bound
MTs and KTs. Thus, we estimated the relative difference of Hecl phosphorylation depending on the paper by Long et al., and the gradient of affinity between poleward and anti-poleward motion. Then we defined a mathematical correlation to adjust our Aurora A interpretation parameter $A u r_{A}$ in order to reproduce the experimental observations of the Aurora A-inhibited cells. Finally, we assumed that cancer cells, even though they are usually overexpressing Aurora A (He et al., 2014; Kao et al., 2017; Umene et al., 2015), behaved analogously, as they showed a comparable Hec1 phosphorylation impairment. The correlation is defined as follows:

$$
F_{g}=\tau e^{-\delta\left(A u r_{A}-A u r_{A o}\right)^{2}}
$$

Where:

$F_{g}=$ Strength gradient between shrinking and growing KT

$\tau=$ Pre-exponential adjustment value

$\delta=$ Coefficient adjustment value

$A u r_{A}=$ Aurora A expression level parameter

$A u r_{A o}=$ Baseline associated Aurora A expression level paramenter 


\section{RESULTS}

The model incorporating Aurora A activity gradient reproduces chromosome oscillations and correction of erroneous KT-MT attachments

We experimentally found that efficient chromosome oscillation and correction of erroneous KT-MT attachments require Aurora A activity, which forms a gradient peaking at spindle poles (Iemura et al., 2021). This is through phosphorylation of Hecl that reduces its affinity to MTs. Therefore, based on previous models, we developed a mathematical model describing chromosome dynamics in which the Aurora A activity gradient regulates the affinity of Hec1 to MTs (Fig. 2F). Our model accurately emulated the chromosome oscillations observed in untreated RPE-1 cells, a non-transformed cell line, during metaphase (Fig. 3A). The kinematics measurements of KT positions clearly showed sister chromatid oscillation (Fig. 3B), and inter-KT distance oscillation (breathing) (Dumont and Mitchison, 2009; Wang et al., 2012; Civelekoglu-Scholey et al., 2013), whose period is one half of that of the sister chromatid oscillation (Fig. 3C). Simultaneously, the rapid jerk of chromosomes, which takes place during the directional change of chromosome motion, was identified as a direct consequence of our KT-MT affinity interpretation (Fig. 3B and D). Furthermore, we were able to faithfully correlate the switching of velocities, caused by the directional instability, with the amount of bound Ndc80 complexes (Fig. 3B and D); whereas the amount of kMTs had no perceptible influence over the change of direction (Fig. 3E).

Regarding the poleward and anti-poleward motion, our model can faithfully replicate the documented experimental observations, which originate the inter-KT oscillation. Based on our results and assumptions, this behavior is due to the KT-MT affinity differences between the paired chromatids, caused by the effect of Aurora A. The leading chromatid is appreciably stalled as it approaches the pole (Fig. 3B), which is a direct consequence of the reduction of the KT-MT affinity (Fig. 3D) and an increasing polar ejection force. In contrast, the KT-MT affinity of the trailing chromatid steadily increases due to reduced Aurora A activity around the spindle equator. In addition, this difference correlates with the already mentioned switching of velocities. Once the anti-poleward motion reaches its peak and undergoes directional instability, the high KT-MT affinity combined with a low polar ejection force as well as a low cohesive viscoelastic force with minimal inter-KT space gives origin to a rapid jerk. Fi- nally, the amount of erroneously-bound KTs peaked at $\mathrm{t}=0$, and decreased rapidly (Fig. 3F). Although erroneous attachments continued to appear at a predefined rate over the time course, they disappeared shortly. Collectively, our mathematical model reproduced chromosome oscillation found in normal cells and exhibited efficient correction of erroneous KTMT attachments.

Aurora A inhibition and upregulation suppress chromosome oscillation and impairs efficient error correction

By modifying the Aurora A expression level, we tried to reproduce the chromosome dynamics of $\mathrm{Au}$ rora A-inhibited cells and Aurora A-upregulated cells, which is commonly seen in cancer (Farag, 2011). Starting from the control, we found that our model shows stable amplitude and frequency of chromosome oscillation, which are approximately $1 \mu \mathrm{m}$ and $100 \mathrm{~s}$, respectively (Fig. 4A), comparable to the observation of RPE-1 cells (Fig. 4B). The tendency of the amount of erroneous attachments shows that they were corrected mainly within the first oscillation cycle (Fig. 4C). Despite erroneous attachments continued to occur, they gradually decreased and were completely eradicated before $300 \mathrm{~s}$ elapsed. Next, we simulated the situation of Aurora A inhibition by excluding the Aurora A activity gradient from the model. Our model of Aurora A inhibition showed a loss of directional instability of sister chromatids and an abnormal inter-KT stretching (approximately $2 \mu \mathrm{m}$; Fig. 4D), which are caused by a lack of Aurora A activity that leads to the stabilization of the KT-MT interactions. This KT behavior is similar to that observed in RPE-1 cells treated with MLN8237, an Aurora A inhibitor (Fig. 4E). Regarding the error correction mechanism, the amount of erroneous attachments decreased initially, a tendency attributable to the overstretching of the inter-KT space, but did not decrease further in the later time points (Fig. 4F). Finally, we tried to reproduce the situation of cancer cells, in which Aurora A is commonly upregulated (He et al., 2014; Kao et al., 2017; Umene et al., 2015). Our model showed an erratic directional instability, as well as an inter-KT stretching (approximately $1 \mu \mathrm{m}$; Fig. 4G), which is similar to the observation in HeLa cells, a cancer-derived cell line (Fig. 4H). Albeit the motion of sister KTs was coordinated, there was little oscillatory or periodical movements between the chromatids. Despite the destabilization of the KT-MT attachments, caused by a steeper Aurora A gradient, the amount of erroneous attachments initially 
A

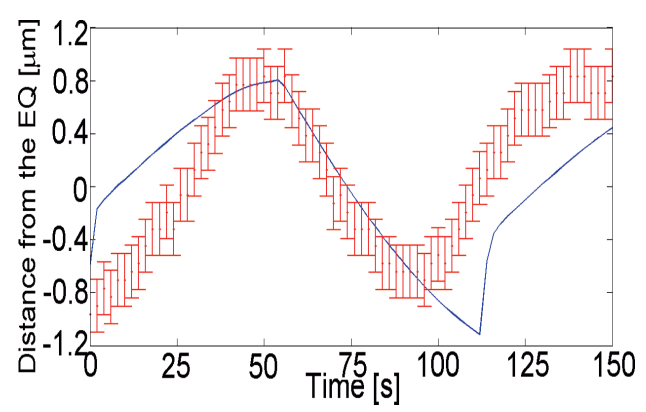

C

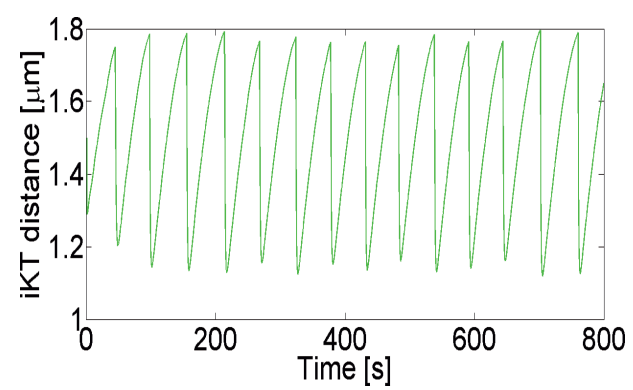

$E$

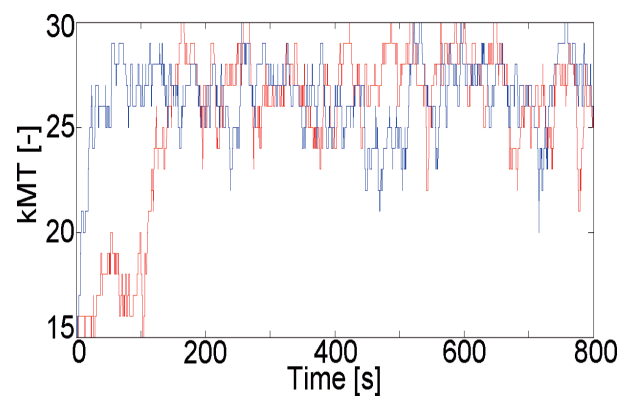

B
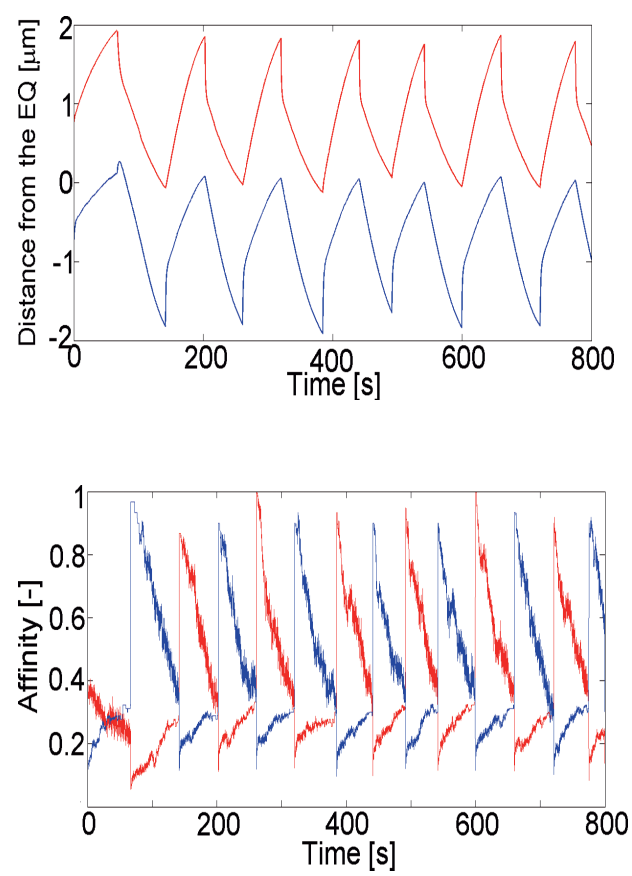

$\mathrm{F}$

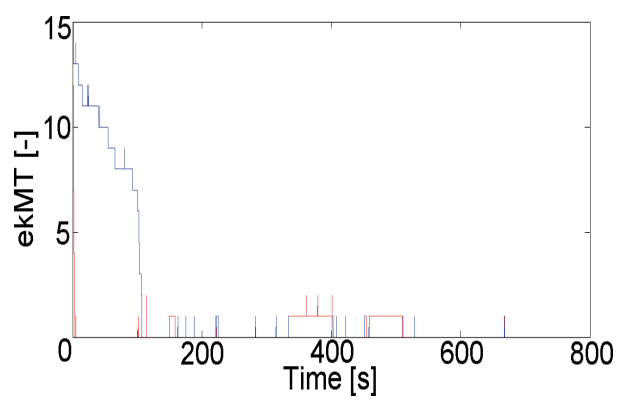

Fig. 3 The uni-dimensional model incorporating Aurora A activity gradient reproduces chromosome oscillations and correction of erroneous KT-MT attachments. (A) Comparison of KT motion obtained by the simulated tracking (blue) with that observed in live imaging of RPE-1 cells (red), shown as distance from the spindle equator (EQ). For the KT motion in RPE-1 cells, the average values from 11 measurements of one of the independent KTs tracked during metaphase are shown. (B) Chromosome oscillation tracking shown as positions of sister KTs (left KT in red and right KT in bule) over time. The position of the spindle equator is set to zero. (C) Inter-KT (iKT) distance over time. (D) Time evolution of the affinity (ratio of attached Ndc80 complexes to a MT compared to the maximum possible amount of Ndc80 attachments) to MTs for the left (red) and right (blue) KTs. (E) Time evolution of the total number of attached MTs (kMT) to the left (red) and right (blue) KTs. (F) Time evolution of the total number of erroneous attachments (ekMT) for the left (red) and right (blue) KTs.

decreased but increased thereafter (Fig. 4I). In summary, these data suggest that suppression of chromosome oscillation and inefficient error correction are seen not only when Aurora A is inhibited, but also when Aurora A is upregulated that is prevalent in cancer cells.
Effects of Aurora A expression level on chromosome oscillation and error correction in the model without sister chromatids rotation (1D interpretation)

In order to test our interpretation of the Aurora A effect on the chromosome kinetics, we looped our model described above, which simulates the motion of sister chromatids only along the spindle axis without rotation (1D interpretation), with the Aurora A activity level as a variable. Considering that our model was able to reproduce the behavior of regular 
A

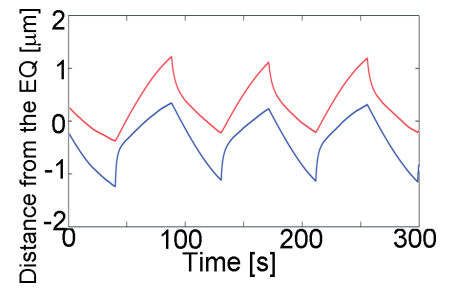

D

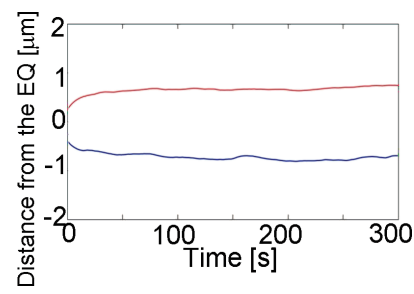

G

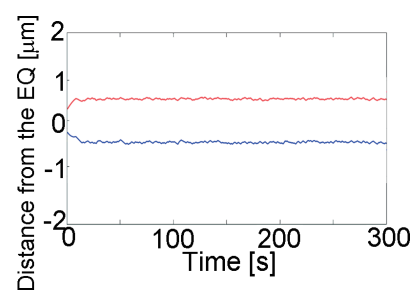

B

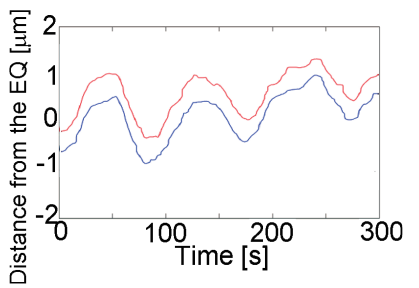

E

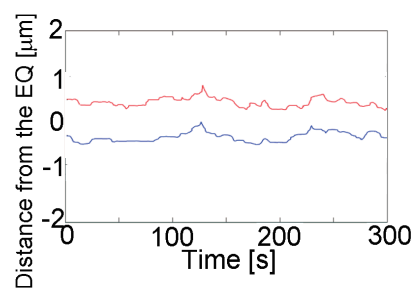

H

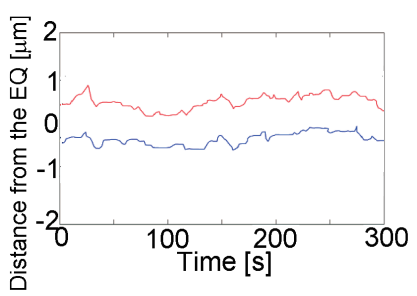

C

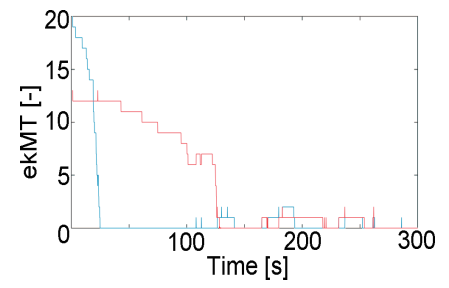

F

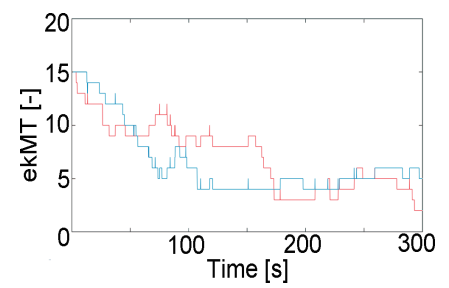

I

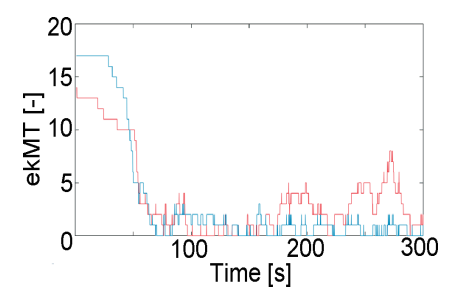

Fig. 4 Aurora A inhibition and upregulation suppress chromosome oscillation and impair efficient error correction. (A) Simulated chromosome oscillation of a cell under standard condition. Positions of sister KTs (left KT in red and right KT in bule), shown as distance from the spindle equator (EQ), over time are shown. (B) Observed chromosome oscillation of an RPE-1 cell. Positions of sister KTs (left KT in red and right KT in bule) over time are shown. (C) Time evolution of the total number of erroneous attachments (ekMT) for the left (red) and right (blue) KTs in a cell under standard condition. (D) Simulated chromosome oscillation of a cell without the Aurora A-like activity gradient. Positions of sister KTs (left KT in red and right $\mathrm{KT}$ in bule) over time are shown. (E) Observed chromosome oscillation of an RPE-1 cell treated with an Aurora A inhibitor (MLN8237). Positions of sister KTs (left KT in red and right KT in bule) over time are shown. (F) Time evolution of the total number of erroneous attachments for the left (red) and right (blue) KTs in a cell without the Aurora A-like activity gradient. (G) Simulated chromosome oscillation of a cell with a higher Aurora A-like activity gradient. Positions of sister KTs (left KT in red and right KT in bule) over time are shown. (H) Observed chromosome oscillation of a HeLa cell. Positions of sister KTs (left KT in red and right KT in bule) over time are shown. (I) Time evolution of the total number of erroneous attachments for the left (red) and right (blue) KTs in a cell with the high Aurora A-like activity gradient.

RPE-1 cells, we determined this configuration as the baseline. In addition, to represent a cancer-like behavior (Bakhoum et al., 2009a, 2009b), we set one group of simulations to emulate highly-stabilized MTs by masking the catastrophe events. The results of the simulations reproduced the tendency observed in our experimental results. The oscillation amplitude peaked around the baseline level and decreased following an asymmetrical Gaussian curve-like behavior (Fig. 5A). Thus, both downregulation and upregulation of Aurora $\mathrm{A}$, in the presence of regular and highly-stabilized MTs, suppress the chromosome oscillation. The oscillation frequency also showed an asymmetrical Gaussian behavior depending on the Aurora A expression level (Fig. 5B). Reduced oscillation amplitude in the simulation of both downregulation and upregulation of Aurora A expression is in agreement with the experimental data of Aurora A-inhibited RPE-1 cells and HeLa cells. The inter-KT distance showed two peaks (Fig. 5C). One is a higher peak when Aurora A is downregulated, and the other is a lower peak when Aurora A is upregulated. As expected, the average amount of proper attachments was bigger when MTs are high- 
A

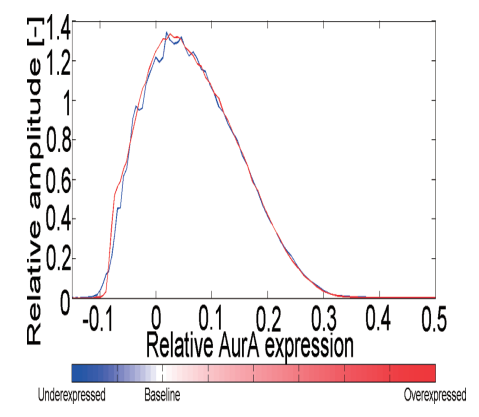

D

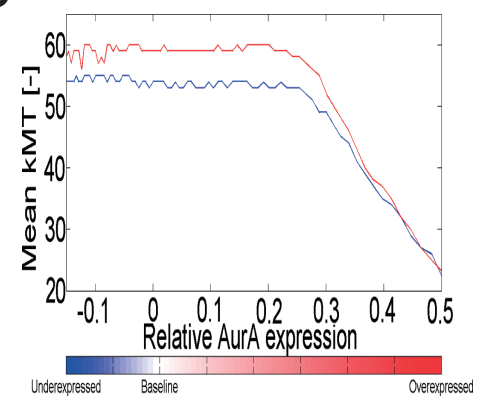

B

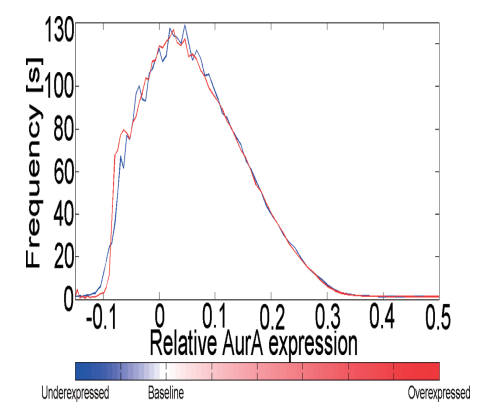

C

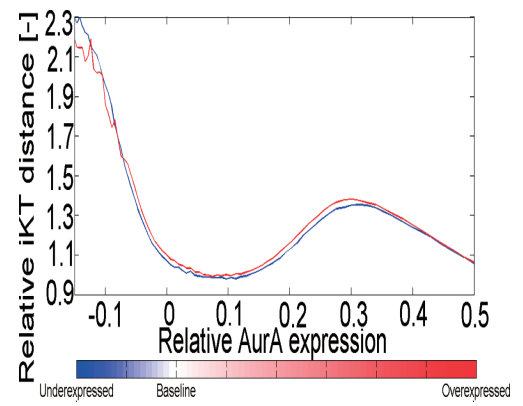

E

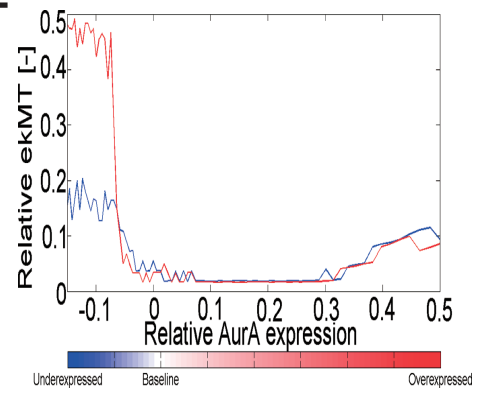

Fig. 5 Effects of Aurora A expression level over the chromosome dynamics and erroneous attachments in uni-dimensional interpretation. (A) Average simulated amplitude of chromosome oscillation relative to the average experimental amplitude value (measured on 11 independent set of KTs on metaphase synchronized RPE-1 cells) over different levels of Aurora A expression. (B) Frequency of chromosome oscillation over different levels of Aurora A expression. (C) Average simulated inter-KT (iKT) distance relative to the average experimental inter-KT value (measured on 11 independent set of KTs on metaphase synchronized RPE-1 cells) over different levels of Aurora A expression. (D) Number of properly attached MTs to a KT (kMT) over different levels of Aurora A expression. (E) Number of erroneous attachments to a KT (ekMT) over different levels of Aurora A expression. Blue represents normal cellular condition, while red represents cellular condition under highly-stabilized MTs (no catastrophe). The horizontal axis represents the base 10 logarithm of the simulated Aurora A expression value, relative to the value that yields the experimental values of amplitude and frequency of chromosome oscillation: the simulated normal Aurora A expression value.

ly-stabilized, but it decreased when Aurora A is upregulated irrespective of MT stability (Fig. 5D). Finally, the average amount of erroneous attachments increased when Aurora A was both downregulated and upregulated (Fig. 5E). Highly-stabilized MTs tend to be more susceptible to erroneous attachments when Aurora A was downregulated. This can be associated with the longevity of the initial attachments, which are randomly set between two equiprobable states (correct/erroneous). In contrast, the difference between regular and highly-stabilized MTs was not apparent when Aurora A was upregulated (Fig. 5E), probably because KT-MT attachment was destabilized in both cases in the current setting of the parameters. Overall, the amplitude and frequency of chromosome oscillation and the efficiency of error correction were reduced not only when Aurora A was downregulated, but also when it was upregulated.
Effects of Aurora A expression level on chromosome oscillation and error correction in the model with sister chromatids rotation ( $2 D$ interpretation)

In order to avoid using an arbitrary constant to reproduce the erroneous attachment occurrence rate, we introduced the sister chromatids rotation in our model (2D interpretation). We looped the $2 \mathrm{D}$ interpretation simulation, with Aurora $\mathrm{A}$ as a variable. By analyzing 3 different expression levels of Aurora A; baseline, 50\% downregulation, and 200\% upregulation of the baseline gradient, we were able to confirm a consistent correlation between the behaviors of Aurora A-downregulated cells and Aurora A-upregulated cells. The initial condition for all the cycles was a completely vertically-oriented chromosome. In normal cells, the orientation of the KTs was corrected in a damped oscillatory tendency, as is shown by the values of angle $\alpha$ (Fig. 6A, Video 1). When the simulation starts, the chromosome tilts 
A

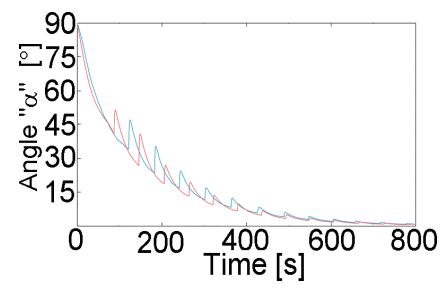

D

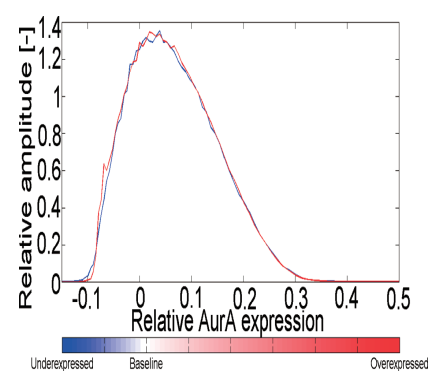

G

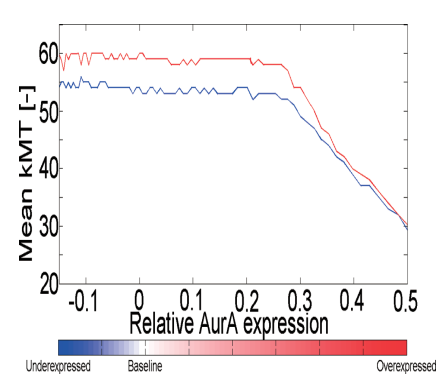

B

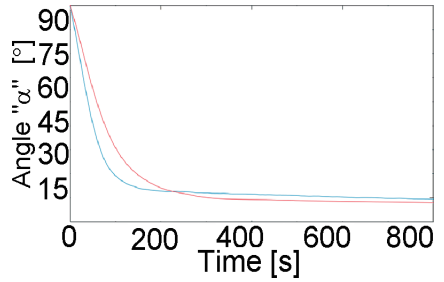

E

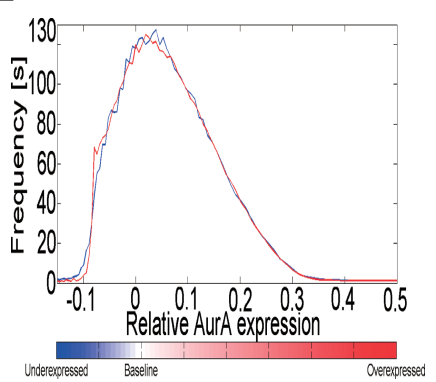

$\mathrm{H}$

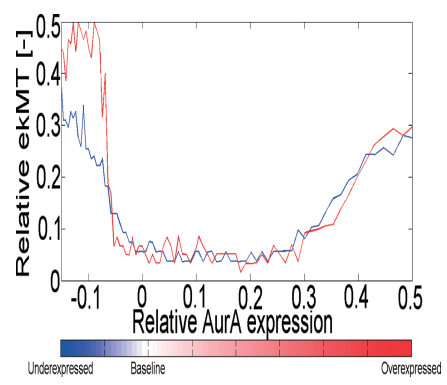

C

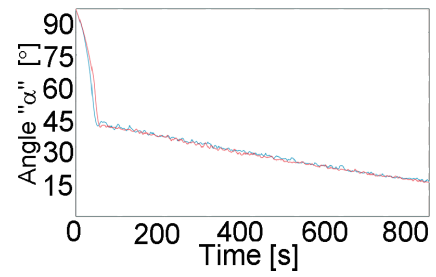

F

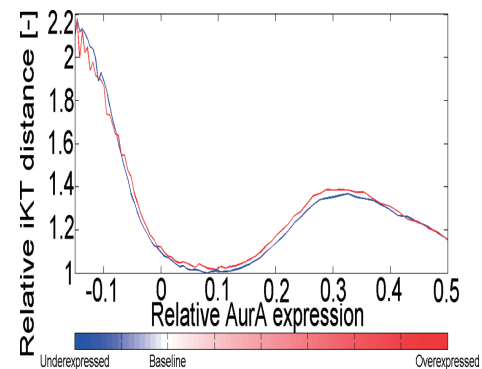

I

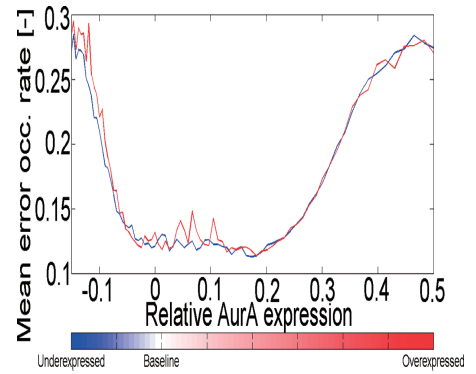

Fig. 6 Effects of Aurora A expression level over the chromosome dynamics and erroneous attachments in bi-dimensional interpretation. (A) Angle of a KT pair in the simulation under baseline Aurora A-like activity. (B) Angle of a KT pair in the simulation without Aurora A-like activity gradient. (C) Angle of a KT pair in the simulation with a higher Aurora A-like activity gradient. (D) Average simulated amplitude of chromosome oscillation relative to average experimental amplitude value over different levels of Aurora A expression. (E) Frequency of chromosome oscillation over different levels of Aurora A expression. (F) Average simulated inter-KT (iKT) distance relative to the average experimental inter-KT value over different levels of Aurora A expression. (G) Number of properly attached MTs to a KT (kMT) over different levels of Aurora A expression. (H) Number of erroneous attachments to a KT (ekMT) over different levels of Aurora A expression. (I) Mean erroneous attachment occurrence ratio of a KT, calculated by dividing the number of erroneous attachments with the sum of proper and erroneous attachments, over different levels of Aurora A expression. In the 1D condition this value is set to a constant, whilst in the $2 \mathrm{D}$ interpretation the value is given by the variable $\beta(t)$. Blue represents normal cellular condition, while red represents cellular condition under highly-stabilized MTs (no catastrophe). As in Fig. 5, the horizontal axis represents the simulated relative Aurora A expression value on a logarithmic scale.

vigorously but this motion progressively subsides. By the end of the simulation ( $800 \mathrm{~s})$, the angle between the chromosome axis and the spindle axis (angle $\alpha$ ) was negligible. Additionally, there was no explicit difference between the regular and highly-stabilized MTs. On the contrary, Aurora A-downregulated and upregulated cells failed to fully eliminate the tilting of the chromosomes (Fig. 6B and $\mathrm{C}$, Video 2 and 3), which resulted in a higher vulnerability to erroneous attachments. On the Aurora A-downregulated condition, the difference between the tendencies of highly stabilized-MTs (red line) and normal MTs (blue line), despite becoming smaller towards the end of the simulation, was appreciable. Considering that Aurora A inhibition leads to more stable KT-MT interactions, the initial conditions regarding the stability of the MTs of the system tend to have a bigger influence over the final 
value of $\alpha$ (Fig. 6B). When Aurora A was upregulated, there was no clear disparity between the value of $\alpha$ yielded by regular and highly-stabilized MTs (Fig. 6C), probably because the difference in MT stability between both conditions was not enough to compensate for the increased effect of up-regulated Aurora A.

The chromosome dynamics obtained from this loop, as the 1D interpretation, correlates to the experimental observations. The amplitude (Fig. 6D) and frequency (Fig. 6E) of oscillation showed no perceivable change from the $1 \mathrm{D}$ model. The inter-KT distance, despite following the same tendency as the 1D interpretation, was held to a slightly smaller maximum value (Fig. 6F). Considering that the chromosome is located on the latitudinal center of the cell, every possible attachment will put the chromatids under vertical compression, which may be the cause of the reduced inter-KT distance in the $2 \mathrm{D}$ interpretation. As in the $1 \mathrm{D}$ interpretation, the average amount of proper attachments was bigger when MTs were highly-stabilized (Fig. 6G). Regarding the amount of erroneous attachments, deviation from the baseline Aurora A expression level, both downregulation and upregulation, led to the increase of erroneous attachments (Fig. $6 \mathrm{H}$ ). The tendency was similar to that in the 1D interpretation, although the amount was higher in the 2D interpretation. Aurora A downregulation caused a bigger amount of erroneous attachments when MTs were highly-stabilized, which may be due to the longevity of the KTMT interactions under this condition. Finally, the prevalence of erroneous attachments was comparable between Aurora A upregulation and downregulation, as well as between regular and highly-stabilized MTs (Fig. 6I).

Overall, it turned out that both models can reproduce the chromosome dynamics of observed cells. However, our bi-dimensional interpretation allows the simulation to behave independently from an arbitrary value of erroneous attachment occurrence rate. The models clearly show the relationship between chromosome oscillation and error correction, which are regulated by Aurora A expression level.

\section{DISCUSSION}

\section{Comparison with previous models}

Our model is based on similar equations in a previous report (Civelekoglu-Scholey et al., 2013) for PtK1 chromosome dynamics. Their simulation is based on a force-balance model that considers the Ndc80 complex as a Kelvin-Voigt type material, which allows the inclusion of the poleward flux on polymerizing MTs and the intrinsic setting of the MT dynamics via tension-dependent relationships. Although these considerations contribute to the exactitude of the results, they make the definition of erroneous attachments difficult, which is why we included a concept introduced by another report (Gay et al., 2012); on-off KT-MT interactions. This means that the KT-MT dynamics are mediated by the Ndc80 complexes with negligible viscoelastic internal forces, and are either attached or detached to the plus end of a MT.

As in the report by Civelekoglu-Scholey et al. (2013), we consider that the $\mathrm{Ndc} 80$ complexes are independent from each other, and hold different levels of affinity for the MT tips, according to their polymerizing/depolymerizing state. We assume that, as it has been reported by Long et al. (2017), MTs in a poleward motion have a higher affinity to KTs (active force), than those in an anti-poleward motion (passive force). Additionally, our Aurora A interpretation regulates the MT attachment-detachment events by the relative position of KTs to the spindle pole, and thus destabilizes the KT-MT interactions as the KT approaches the pole it is oriented to. As reported by Civelekoglu-Scholey et al. (2013), the bond between a polymerizing MT and a KT is considered weak while that between a depolymerizing MT and a KT strong. Albeit the MT dynamics are strictly defined as position-dependent, the forces that drive the attachment/detachment events described by Civelekoglu-Scholey et al. (2013) are also calculated in our model.

In another model proposed by Gay et al. (2012), it is considered that $\mathrm{KT}$ is a Kelvin-Voigt solid and KT-MT interactions have a switch like behavior. This model was able to reproduce the chromosome dynamics during metaphase, as longs as the tug-ofwar equilibrium is tilted towards directional instability by an intrinsic and independent variable within the system. In their simulation, the mechanism that triggers directional instability is a position-dependent autocorrelation, whereas in our model, it is a deterministic law based on the position-dependent interpretation of Aurora A, which is also considered as the main contributor to the correction of erroneous attachments. In contrast, Gay et al. (2012) focused on Aurora B as the error correction mechanism, which functions in an inter-KT-distance-dependent manner.

Regardless of the purpose or approach, the similarity between their results and ours corroborate the veracity of our interpretation of the phenomenon. 
Our data suggest that in the central region of the spindle, the pulling force associated with the poleward motion is bigger than its anti-poleward counterpart, although the former declines as the chromosome approaches the spindle pole. In this area, the poleward-associated force is overcome by its steadily increasing counterpart, and consequently, directional instability is triggered, which switches the roles from leading to trailing. The action of switching embeds a KT-MT affinity gradient that directly affects the forces engaged in this tug-of-war, and decreases as a KT approaches the spindle pole. It is of note, however, two Hecl phosphorylation sites, serine 55 (S55) and 69 (S69), which are mainly responsible for chromosome oscillation, are differentially regulated; while Hec1-S55 is phosphorylated by Aurora A localizing to the spindle, Hec1-S69 is mainly phosphorylated by Aurora A residing at inner centromere and only partially responds to chromosome oscillation (DeLuca et al., 2018; Iemura et al., 2021). Therefore, although Hec1 phosphorylation by Aurora A is required for efficient chromosome oscillation, to what extent the Aurora A activity gradient along the spindle drives oscillatory motion warrants further investigation. In a physiological condition, it may work together with other mechanisms, such as force-dependent detachment kinetics of the $\mathrm{Ndc} 80$ complex and length- and polymerization rate-dependent catastrophe frequency of MTs, which were described in the previous report (Civelekoglu-Scholey et al., 2013).

\section{Aurora A, chromosome oscillation, and error cor- rection}

Whether the main cause of chromosome oscillation is a gradient of KT-MT affinity or the effect of MTs dynamics remains unknown (Kops et al., 2010). In addition, although an increasing number of researches have linked perturbations of chromosome dynamics with missegregation and CIN, details of this mechanism are yet to be specified. As Aurora A irregularities are associated with error correction deficiency and CIN, developing mathematical models that provide an insight into their possible roles is of significance. Moreover, understanding how these irregularities are manifested on measurable variables could help establishing new protocols for future experiments. Our results suggest that the presence of a KT-MT destabilizing gradient allows, not only to replicate the oscillatory motion observed in regular cells, but also to establish a framework to understand the role of Aurora A as a significant piece in the error correction mechanism during metaphase.
As a consequence of modifying the Aurora A expression level, we were able to reproduce chromosome dynamics comparable to the observations of Aurora A-inhibited RPE-1 cells, and HeLa cells. Our results coincide with the experimental observations that link Aurora A expression irregularities with abnormal chromosome dynamics (Zhang et al., 2008; He et al., 2014; Umene et al., 2015; DeLuca et al., 2018; Iemura et al., 2021). The latter have been found to be caused by defective KT-MT interactions (Bakhoum et al., 2009a; Amaro et al., 2010). Considering that the role of Aurora A in our model is directly related to the KT-MT attachments, and that the deviation from its baseline expression leads to the disruption of normal chromosome oscillations, our interpretation is considered to be consistent with previously reported experimental results. However, the caveat is that in cancer cell lines we observed in a recent report, the upregulation of Aurora A was not apparent even though they showed attenuated chromosome oscillation (Iemura et al., 2021). Further study is required to specify the main cause of attenuated chromosome oscillation in cancer cells.

\section{Uni-dimensional and bi-dimensional interpretation}

In this research, we evaluated two different interpretations of the chromosome dynamics during metaphase: one without sister chromatids rotation (uni-dimensional interpretation) and one with sister chromatids oscillation (bi-dimensional interpretation). The uni-dimensional interpretation relies on a fixed value of erroneous attachment occurrence probability, which is an approach that has been used in a previous model (Gay et al., 2012). On the other hand, in the bi-dimensional model, the erroneous attachment occurrence rate becomes an intrinsic variable of the system by including two additional degrees of freedom into the model (vertical translation and rotation). The definition of the erroneous attachment occurrence rate is based on a geometric approach to KT-MT interaction; completely bi-oriented chromosomes have a negligible probability of attaching to the diametrically "incorrect" spindle pole, and vertically-oriented chromosomes have an equal probability of connecting to the either spindle pole (Stumpff and Asbury, 2008). To show the robustness of our model, we started the simulation with a completely vertically-oriented chromosomes, an extreme situation. Our simulation results suggest that even when similar number of MTs attach to a single KT from both spindle poles (balanced-merotelic attachment [Gregan et al., 2011]), oscillation amplitude gradually increases while merotelic at- 
tachments are resolved. In a physiological condition, however, chromosomes are usually oriented horizontally relative to the spindle axis due to congression through lateral attachment, making bi-orientation establishment efficient (Tanaka, 2012; Iemura and Tanaka, 2015; Itoh et al., 2018; Kuniyasu et al., 2018). Our results collate to the experimental observations (Magidson et al., 2011), and other simulated tendencies that link bi-orientation with error correction efficiency (Paul et al., 2009). The model was able to reproduce the transition from a vertically oriented position to a spindle-axis-parallel position, within the average duration of metaphase in a normal cell, as well as the inability of abnormal cells (deviations from the Aurora A baseline) to become completely horizontally oriented (Kitajima et al., 2011).

Through the evaluation of Aurora A effect on the error correction mechanism, we discovered that Aurora $\mathrm{A}$ expression level has to be in a proper range to ensure efficient correction of erroneous attachments. Our results limit the range within which erroneous attachments are efficiently eliminated between approximately $80 \%$ and $180 \%$ (approximately -0.1 and 0.25 respectively, on a base $10 \log$ arithmic scale) of the Aurora A expression level used to reproduce the oscillation observed in normal cells. Despite these values are merely a stipulation of what the real range could be, our model suggests that Aurora A inhibition as well as its upregulation, like the condition observed in cancer cells (Ertych et al., 2014; He et al., 2014; Nicholson et al., 2015; Kao et al., 2017), leads to deficient erroneous attachment correction, and consequently to chromosome missegregation and CIN. Although further considerations and new parameters will fade the gap between simulation and reality, our model provides a framework to understand the relevance of Aurora A expression level on the error correction mechanism and chromosome dynamics.

\section{VIDEO INFORMATION}

Video 1 Simulation of KT-MT attachment of a sister KT pair in 2D interpretation in normal condition. Inter-KT distance is shown below.

Video 2 Simulation of KT-MT attachment of a sister KT pair in 2D interpretation when Aurora A is downregulated. Inter-KT distance is shown below.

Video 3 Simulation of KT-MT attachment of a sister KT pair in 2D interpretation when Aurora A is upegulated. Inter-KT distance is shown below.

\section{Acknowledgements}

We thank H. Hochegger for RPE-1 cell line, and T. Hirota for HeLa Kyoto cell line. We also thank members of the K.T. laboratory for discussions, and A. Harata for technical assistance. This work was supported by JSPS KAKENHI Grant Numbers $16 \mathrm{H} 06635$ and $18 \mathrm{~K} 15234$, grants from Uehara Memorial Foundation, Kanae Foundation for the Promotion of Medical Science, and Gonryo Medical Foundation to K. I., and JSPS KAKENHI Grant Numbers 16K14604 and 18H02434, and a grant from the Takeda Science Foundation to K.T.

\section{CONFLICT OF INTEREST}

The authors declare no competing financial interests.

\section{REFERENCES}

Abe Y, Sako K, Takagaki K, Hirayama Y, Uchida KS, et al. (2016) HP1-Assisted Aurora B Kinase Activity Prevents Chromosome Segregation Errors. Dev Cell 36, 487-497.

Amaro AC, Samora CP, Holtackers R, Wang E, Kingston IJ, et al. (2010) Molecular control of kinetochore-microtubule dynamics and chromosome oscillations. Nat Cell Biol 12, 319329.

Asbury CL (2017) Anaphase A: Disassembling microtubules move chromosomes toward spindle poles. Biology (Basel) 6 , 15.

Bakhoum SF and Compton DA (2012) Chromosomal instability and cancer: a complex relationship with therapeutic potential. J Clin Invest 122, 1138-1143.

Bakhoum SF, Genovese G and Compton DA (2009a) Deviant kinetochore microtubule dynamics underlie chromosomal instability. Curr Biol 19, 1937-1942.

Bakhoum SF, Thompson SL, Manning AL and Compton DA (2009b) Genome stability is ensured by temporal control of kinetochore-microtubule dynamics. Nat Cell Biol 11, 27-35.

Brouhard GJ and Hunt AJ (2005) Microtubule movements on the arms of mitotic chromosomes: polar ejection forces quantified in vitro. Proc Natl Acad Sci USA 102, 13903-13908.

Cimini D (2008) Merotelic kinetochore orientation, aneuploidy, and cancer. Biochim Biophys Acta 1786, 32-40.

Civelekoglu-Scholey G and Cimini D (2014) Modelling chromosome dynamics in mitosis: a historical perspective on models of metaphase and anaphase in eukaryotic cells. Interface Focus 4, 20130073.

Civelekoglu-Scholey G, He B, Shen M, Wan X, Roscioli E, et al. (2013) Dynamic bonds and polar ejection force distribution explain kinetochore oscillations in PtK1 cells. $J$ Cell Biol 201, 577-593.

DeLuca KF, Meppelink A, Broad AJ, Mick JE, Peersen OB, et al. (2018) Aurora A kinase phosphorylates Hec1 to regulate metaphase kinetochore-microtubule dynamics. J Cell Biol 217, 163-177.

Dumont S and Mitchison TJ (2009) Force and length in the mitotic spindle. Curr Biol 19, R749-761.

Ertych N, Stolz A, Stenzinger A, Weichert W, Kaulfuss S, et al. (2014) Increased microtubule assembly rates influence chro- 
mosomal instability in colorectal cancer cells. Nat Cell Biol 16, 779-791.

Farag SS (2011) The potential role of Aurora kinase inhibitors in haematological malignancies. Br J Haematol 155, 561-579.

Gay G, Courtheoux T, Reyes C, Tournier S and Gachet Y (2012) A stochastic model of kinetochore-microtubule attachment accurately describes fission yeast chromosome segregation. $J$ Cell Biol 196, 757-774.

Gordon DJ, Resio B and Pellman D (2012) Causes and consequences of aneuploidy in cancer. Nat Rev Genet 13, 189203.

Goshima G and Scholey JM (2010) Control of mitotic spindle length. Annu Rev Cell Dev Biol 26, 21-57.

Gregan J, Polakova S, Zhang L, Tolic-Norrelykke IM and Cimini D (2011) Merotelic kinetochore attachment: causes and effects. Trends Cell Biol 21, 374-381.

He S, Feng M, Liu M, Yang S, Yan S, et al. (2014) P21-activated kinase 7 mediates cisplatin-resistance of esophageal squamous carcinoma cells with Aurora-A overexpression. PLoS One 9, e113989.

Hong W, Takshak A, Osunbayo O, Kunwar A and Vershinin M (2016) The effect of temperature on microtubule-based transport by cytoplasmic dynein and kinesin-1 motors. Biophys $J$ 111, 1816.

Iemura K, Natsume T, Maehara K, Kanemaki MT, and Tanaka K (2021) Chromosome oscillation promotes Aurora A-dependent Hecl phosphorylation and mitotic fidelity. $J$ Cell Biol 220, e202006116.

Iemura K and Tanaka K (2015) Chromokinesin Kid and kinetochore kinesin CENP-E differentially support chromosome congression without end-on attachment to microtubules. Nat Commun 6, 6447.

Itoh G, Ikeda M, Iemura K, Amin MA, Kuriyama S, et al. (2018) Lateral attachment of kinetochores to microtubules is enriched in prometaphase rosette and facilitates chromosome alignment and bi-orientation establishment. Sci Rep 8, 3888.

Jaqaman K, King EM, Amaro AC, Winter JR, Dorn JF, et al. (2010) Kinetochore alignment within the metaphase plate is regulated by centromere stiffness and microtubule depolymerases. J Cell Biol 188, 665-679.

Kao YT, Wu CH, Wu SY, Lan SH, Liu HS, et al. (2017) Arsenic treatment increase Aurora-A overexpression through E2F1 activation in bladder cells. BMC Cancer 17, 277.

Ke K, Cheng J and Hunt AJ (2009) The distribution of polar ejection forces determines the amplitude of chromosome directional instability. Curr Biol 19, 807-815.

Kinoshita K, Arnal I, Desai A, Drechsel DN and Hyman AA (2001) Reconstitution of physiological microtubule dynamics using purified components. Science 294, 1340-1343.

Kitajima TS, Ohsugi M and Ellenberg J (2011) Complete kinetochore tracking reveals error-prone homologous chromosome biorientation in mammalian oocytes. Cell 146, 568-581.

Kops GJ, Saurin AT and Meraldi P (2010) Finding the middle ground: how kinetochores power chromosome congression. Cell Mol Life Sci 67, 2145-2161.

Kuniyasu K, Iemura K and Tanaka K (2018) Delayed chromosome alignment to the spindle equator increases the rate of chromosome missegregation in cancer cell lines. Biomolecules $9,10$.

Long AF, Udy DB and Dumont S (2017) Hec1 tail phosphorylation differentially regulates mammalian kinetochore coupling to polymerizing and depolymerizing microtubules. Curr Biol
27, 1692-1699 e1693.

Magidson V, O'Connell CB, Loncarek J, Paul R, Mogilner A, et al. (2011). The spatial arrangement of chromosomes during prometaphase facilitates spindle assembly. Cell 146, 555567.

Nicholson JM, Macedo JC, Mattingly AJ, Wangsa D, Camps J, et al. (2015) Chromosome mis-segregation and cytokinesis failure in trisomic human cells. Elife 4, e05068.

Paul R, Wollman R, Silkworth WT, Nardi IK, Cimini D, et al. (2009) Computer simulations predict that chromosome movements and rotations accelerate mitotic spindle assembly without compromising accuracy. Proc Natl Acad Sci USA 106, 15708-15713.

Schindelin J, Arganda-Carreras I, Frise E, Kaynig V, Longair M, et al. (2012) Fiji: an open-source platform for biological-image analysis. Nat Methods 9, 676-682.

Skibbens RV, Skeen VP and Salmon ED (1993) Directional instability of kinetochore motility during chromosome congression and segregation in mitotic newt lung cells: a push-pull mechanism. J Cell Biol 122, 859-875.

Stumpff J and Asbury CL (2008) Chromosome bi-orientation: Euclidian euploidy. Curr Biol 18, R81-83.

Tanaka K (2012) Dynamic regulation of kinetochore-microtubule interaction during mitosis. J Biochem 152, 415-424.

Tanaka K (2013) Regulatory mechanisms of kinetochore-microtubule interaction in mitosis. Cell Mol Life Sci 70, 559-579.

Tanaka K and Hirota T (2016) Chromosomal instability: A common feature and a therapeutic target of cancer. Biochim Biophys Acta 1866, 64-75.

Tanaka TU, Stark MJ and Tanaka K (2005) Kinetochore capture and bi-orientation on the mitotic spindle. Nat Rev Mol Cell Biol 6, 929-942.

Thevenaz P, Ruttimann UE and Unser M (1998) A pyramid approach to subpixel registration based on intensity. IEEE Trans Image Process 7, 27-41.

Umene K, Yanokura M, Banno K, Irie H, Adachi M, et al. (2015) Aurora kinase A has a significant role as a therapeutic target and clinical biomarker in endometrial cancer. Int $J$ Oncol 46, 1498-1506.

Wan X, Cimini D, Cameron L A and Salmon ED (2012) The coupling between sister kinetochore directional instability and oscillations in centromere stretch in metaphase PtK1 cells. Mol Biol Cell 23, 1035-1046.

Wang X, Zhuang X, Cao D, Chu Y, Yao P, et al. (2012) Mitotic regulator SKAP forms a link between kinetochore core complex KMN and dynamic spindle microtubules. $J$ Biol Chem 287, 39380-39390.

Wimbish RT and DeLuca JG (2020) Hec1/Ndc80 tail domain function at the kinetochore-microtubule interface. Front Cell Dev Biol 8, 43.

Zaytsev AV, Mick JE, Maslennikov E, Nikashin B, DeLuca JG, et al. (2015) Multisite phosphorylation of the NDC80 complex gradually tunes its microtubule-binding affinity. $\mathrm{Mol}$ Biol Cell 26, 1829-1844.

Zaytsev AV, Sundin LJ, DeLuca KF, Grishchuk EL and DeLuca JG (2014) Accurate phosphoregulation of kinetochore-microtubule affinity requires unconstrained molecular interactions. $J$ Cell Biol 206, 45-59.

Zhang X, Ems-McClung SC and Walczak CE (2008) Aurora A phosphorylates MCAK to control ran-dependent spindle bipolarity. Mol Biol Cell 19, 2752-2765. 